\title{
EVALUATION OF APICAL BACTERIAL LEAKAGE OF THREE DIFFERENT OBTURATION SYSTEMS AFTER USING TWO DIFFERENT IRRIGATING SOLUTIONS: AN IN VITRO STUDY
}

\author{
Hayam Y. Hassan ${ }^{*}$, Ahmed M. Negm ${ }^{* *}$ and Abeer E. Elsayed ${ }^{* * *}$
}

\begin{abstract}
Aim: The present study aimed to compare between apical bacterial leakage of obturating systems such as EndoRez, ActiV GP and AH Plus sealers using gutta-percha, with two different irrigating materials Biopure MTAD (mixture of doxycycline, acetic acid and detergent) and $2.25 \%$ sodium hypochlorite $(\mathrm{NaOCl})$.
\end{abstract}

Methods: Seventy extracted human mandibular premolars with single canal were divided into two equal groups of 30 samples each according to the irrigating solution used either Biopure MTAD or $2.25 \% \mathrm{NaOCL}$. The other 10 teeth (5 roots positive group and 5 roots negative group) were used as control. The main groups were subdivided into 3 subgroups according to the obturation system used, EndoRez, ActiV GP or AH Plus with gutta percha. Sealing ability was assessed using Enterococcus faecalis (E. faecalis) in a double chamber leakage model. Samples were monitored weekly for periods of 1,4 and 8 weeks to assess the turbidity of the medium in contact with the lower coronal portion of the root segment.

Results: Showed statistically significant increase in the mean apical leakage with specimens irrigated with $2.25 \% \mathrm{NaOCL}$ than Biopure MTAD in all periods, while roots obturated with EndoRez showed less statistically significant leakage than the rest of the experimental materials at 1 and 4 weeks periods while, at 8 weeks period all the experimental materials showed leakage with no statistically significance difference.

Conclusion: EndoRez when used after Biopure MTAD irrigation provides better sealing with root canal dentin.

KEYWORDS: Bacterial leakage, root canal filling, smear layer.

\footnotetext{
* Assistant Professor of Endodontic, Faculty of Dentistry, Suez Canal University, Ismailia, Egypt ** Assistant Professor of Endodontic, Faculty of Dentistry, Ahram Canadian University, Giza, Egypt.

*** Professor of Medical Microbiology \& Immunology, Faculty of Medicine, Suez Canal University, Ismailia, Egypt
} 


\section{INTRODUCTION}

One of the most important goals in endodontic is the creation of hermetic seal using an inert filling material ${ }^{(1)}$. According to Branstetter and VonFraunhofer, if apical foramen is incompletely sealed, apical percolation of blood-borne proteins can cause periapical inflammation as it stagnates and decompose within the canal space. $58 \%$ of reported endodontic failures are due to inadequate obturation of root canals ${ }^{(2,3)}$. New filling materials and techniques were advanced to improve the quality of the root canal seal.

Sealers such as AH Plus which is an epoxy based resin offers very good properties biologically and physically as well as adequate apical sealing ${ }^{(4)}$.

Many recent studies have shown promising results with methacrylate-based resins ${ }^{(5-7)}$. A urethane dimethacrylate resin based sealer EndoRez has become an effective sealer ${ }^{(8)}$ as it works in moist environment due to its hydrophilic nature allowing deeper penetration into dentin but not make that with gutta-percha ${ }^{(8)}$. No adhesion with gutta-percha which creates a weak point as leakage path. In order to create a bond between (sealer-dentin interface) and (sealer-gutta-percha interface), gutta-percha cones covered with methacrylate resin (EndoRez Points) have recently been introduced ${ }^{(9)}$.

ActiV GP precision obturation system is a glassionommer (GI) constructed obturation system. This product is superior to previously (GI) used systems in relation to the working time, radiopacity and handling characteristics ${ }^{(10)}$. In order to overcome the disadvantages of inadequate bonding between (GI) and gutta percha (GP), GI particles have been incorporated inside gutta percha cone and covered its surface as a coating ${ }^{(10)}$.

This study aimed to compare between apical bacterial leakage of obturating systems such as EndoRez, ActiV GP and AH Plus sealer with gutta-percha using two different irrigating materials Biopure MTAD and $2.25 \%$ sodium hypochlorite $(\mathrm{NaOCl})$.

\section{MATERIALS AND METHODS}

Ethical clearance was taken before starting the study. Seventy extracted human mandibular premolars with single root were collected. They were caries free and each had single canal with mature apex. Teeth with cracks or fracture lines were eliminated after examination using loops. Radiographs were taken to ensure that the teeth were with single canaled. The teeth were cleaned of debris and stored in saline solution.

The crowns of the teeth were removed to obtain uniform working length of $17 \mathrm{~mm}$ using a diamond disk (D\&Z, Darmstadt, Germany). The root canals were accessed and mechanically shaped by Race rotary NiTi files (FKG Dentaire, Switzerland) and enlarged to size 40 , taper 0.04 . All the root canals were irrigated with $1.3 \% \mathrm{NaOCl}$ irrigation. Five roots were left without obturation for the positive control group. Other five roots were kept without obturation and the apices were sealed with pink wax for the negative control group. The remaining roots were divided into two main groups $(n=30)$ according to the final irrigation used.

For group 1: Biopure MTAD (Dentsply Tulsa Dental Specialties, Tulsa, OK).

For group 2: $2.5 \% \mathrm{NaOCl}$ irrigation (Oxford Laboratory, Mumbai, India)

Each main group was subdivided into three subgroups $(n=10)$ according to the obturating material used.

Subgroup (1): EndoRez obturating system (Ultradent, South Jordan, Utah, USA.) was used according to the manufacturer's directions. The master cone (40/04) was covered with EndoRez sealer and introduced to full working length. Then, light cured for 40 seconds and excess of cone was cut.

Subgroup (2): A single-cone ActiV GP obturating system (Brasseler USA, Savannah, GA) was used as the manufacturer's directions. The master cone (40/04) was covered with ActiV GP sealer and introduced to full working length and excess of cone 
was cut. Layer of ActiV GP sealer was deposited on top of the master cone to create a coronal seal.

Subgroup (3): AH Plus sealer plus gutta-percha cones were used for obturation (Dentsply, Konstanz, Germany) according to manufacturer's directions. The master cone (40/04) was covered with sealer and introduced to full working length. A lateral compaction technique was used with auxiliary cones and excess of cones were cut.

Three layers of nail polish coated the whole root surface except the apical $2 \mathrm{~mm}$. Teeth were kept in incubator for one week at $37^{\circ} \mathrm{C}$ and $100 \%$ humidity. $10 \mathrm{~mm}$ of the end portion of blue tips were cut off with hot spatula. The blue tip end was fixed to the apical portion of each root. The junction of the root and the blue tip was sealed with light cured flowable composite (3M ESPE, USA).

This study has been already done under a laminar flow hood. For detection of apical bacterial leakage, a strain of Enterococcus faecalis (ATCC 29212) was used as a bacterial marker. 24 hours colonies of E. faecalis were cultured in $20 \mathrm{ml}$ of Trypticas soy broth (TSB) (Oxoid Ltd, Basingstoke, United Kingdom) and incubated for 24 hours until the optical density of the bacterial suspension was adjusted to $3 \times 10^{8} \mathrm{CFU} / \mathrm{ml}$, by comparing its turbidity to 1 McFarland standard. The external surface of each sample was disinfected by $5 \mathrm{ml}$ of $5.25 \%$ sodium hypochlorite and washed with $20 \mathrm{ml}$ of sterile distilled water. About $0.5 \mathrm{ml}$ sterile (TSB) was added in each sterile Wassermann tube and each tooth was immersed in the tube, till the coronal portion about $2 \mathrm{~mm}$ of the roots were immersed in the broth. 1 $\mathrm{ml}$ of the previously prepared bacterial suspension was applied into the blue tip sealed to the apical root portion using an automatic micropipette. The Wassermann tubes containing the samples were perfectly closed then labeled and numbered according to group and subgroup, held vertically within its rack. All the samples were incubated at $37^{\circ} \mathrm{C}$ and $100 \%$ humidity and checked after one week, 4 and 8 weeks. Leakage was detected by turbidity of the
(TSB) medium in contact with the coronal portion of the root segment. Every 7 days during the experimental period, the (TSB) inoculated with E. faecalis was replaced with a new $1 \mathrm{ml}$ of freshly prepared bacterial suspension.

Sterile plates containing IBB-Enterococcus Agar (Difco Microbiology, Inc. USA) were prepared and labeled corresponding to the number of the Wassermann tubes. $10 \mu \mathrm{l}$ from the turbid (TSB) medium was transferred to sterile plate and incubated at $37^{\circ} \mathrm{C}$ and $100 \%$ humidity for 48 hours. Bacterial viability was detected by black color due to growth of bacteria. All the data obtained were recorded and statistically analyzed.

\section{RESULTS}

Kruskal-Wallis multiple comparison statistical analysis test compared the apical bacterial leakage of the two irrigants and the three obutrating systems at the three different time intervals as shown in (Table 1) and Fig. (1).

After one week, Biopure MTAD did not show any bacterial leakage with any obturating system and recorded the same mean value (15.5). But $2.5 \%$ sodium hypochlorite revealed apical bacterial leakage with no significant difference among the three obturating systems $(\mathrm{P}<0.05)$.

After 4 weeks, there was a significant difference recorded for the three subgroups using Biopure MTAD irrigant $(\mathrm{P}<0.05)$. EndoRez recorded the least apical bacterial leakage mean value (12). While, ActiV GP and AH Plus recorded higher apical bacterial leakage mean values $(15,19)$. Specimens of $2.5 \%$ sodium hypochlorite irrigant revealed different apical bacterial leakage mean values (11, 17, and 18.5). There was a significant difference found among the three subgroups $(\mathrm{P}<0.05)$.

After 8 weeks, all the specimens in all subgroups markedly increased mean values of apical bacterial leakage, yet no significant difference was found between them $(\mathrm{P}<0.05)$. 
TABLE (1): The Mean Rank values of apical bacterial leakage of the two irrigants and the three obutrating systems at the three different time intervals.

\begin{tabular}{|c|c|c|c|c|c|}
\hline Irrigant Solutions & Time Intervals & Obturating Systems & Mean Rank ${ }^{1}$ & Chi-Square value & P value \\
\hline \multirow{9}{*}{ 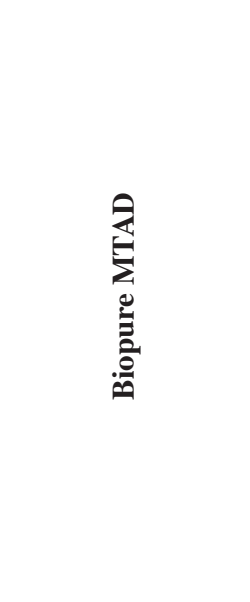 } & \multirow{3}{*}{1 Week } & EndoRez & 15.5 & \multirow{3}{*}{0.0001} & \multirow{3}{*}{1.000} \\
\hline & & ActiV GP & 15.5 & & \\
\hline & & AH Plus & 15.5 & & \\
\hline & \multirow{3}{*}{4 weeks } & EndoRez & 12 & \multirow{3}{*}{6.845} & \multirow{3}{*}{0.033} \\
\hline & & ActiV GP & 15 & & \\
\hline & & AH Plus & 19 & & \\
\hline & \multirow{3}{*}{8 weeks } & EndoRez & 11.5 & \multirow{3}{*}{4.640} & \multirow{3}{*}{0.098} \\
\hline & & ActiV GP & 17.5 & & \\
\hline & & AH Plus & 17.5 & & \\
\hline \multirow{9}{*}{ 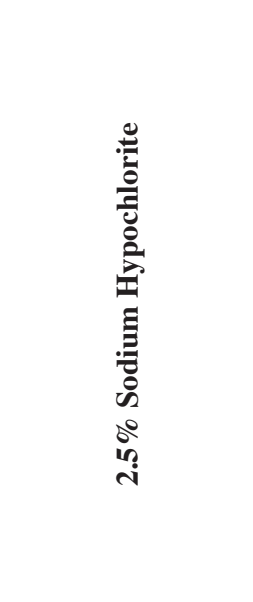 } & \multirow{3}{*}{1 Week } & EndoRez & 12.5 & \multirow{3}{*}{5.413} & \multirow{3}{*}{0.067} \\
\hline & & ActiV GP & 14 & & \\
\hline & & AH Plus & 20 & & \\
\hline & \multirow{3}{*}{4 weeks } & EndoRez & 11 & \multirow{3}{*}{8.458} & \multirow{3}{*}{0.015} \\
\hline & & ActiV GP & 17 & & \\
\hline & & AH Plus & 18.5 & & \\
\hline & \multirow{3}{*}{8 weeks } & EndoRez & 13.5 & \multirow{3}{*}{4.143} & \multirow{3}{*}{0.126} \\
\hline & & ActiV GP & 16.5 & & \\
\hline & & AH Plus & 16.5 & & \\
\hline
\end{tabular}

${ }^{1}$ The Kruskal Wallis test (one-way analysis of variance by ranks), $(P<0.05)$.

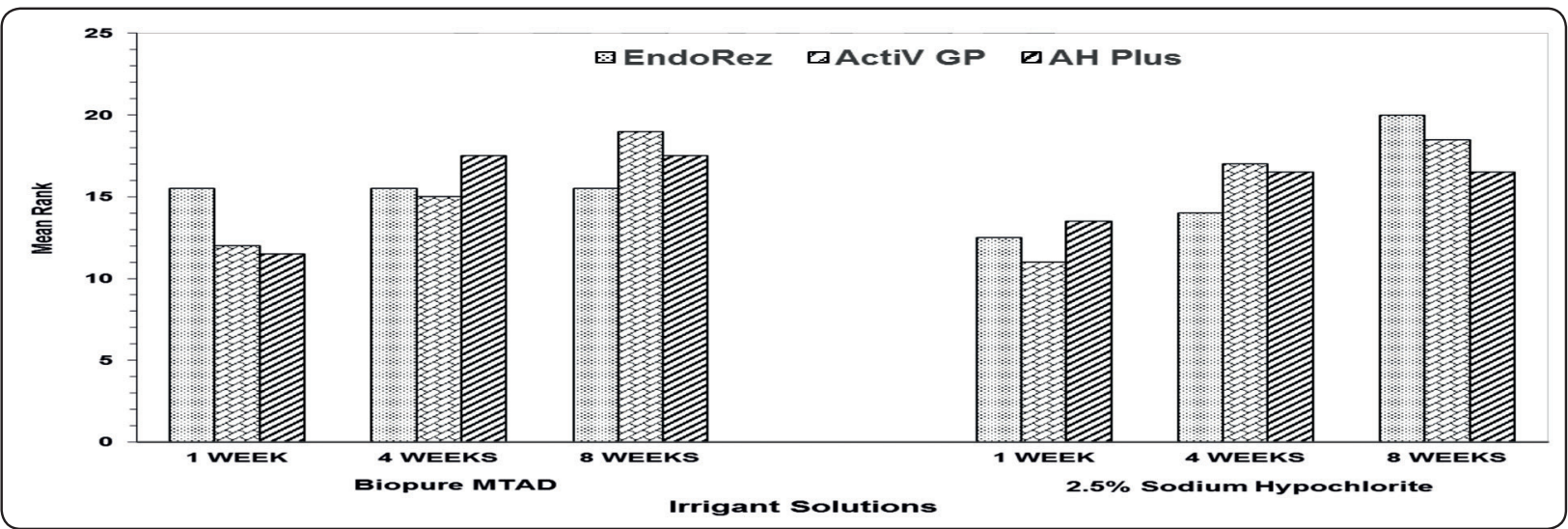

Fig. (1): The Mean Rank values of apical bacterial leakage of the two irrigants and the three obutrating systems at the three different time intervals. 


\section{DISCUSSION}

Successful results in endodontic treatment are achieved by proper sealing of the root canal ${ }^{(11)}$. Inadequate apical and coronal seal is the most important reason for endodontic treatment failure ${ }^{(12)}$.

In this study apical bacterial leakage was compared among the sealing of ActiV GP EndoRez and AH Plus sealers with gutta percha using Biopure MTAD and $2.5 \%(\mathrm{NaOCl})$ as irrigating solutions at 1,4 and 8 weeks period intervals.

Sealing properties of endodontic materials using leakage studies constitutes an important area of research ${ }^{(13)}$. Different approaches have been used to determine the sealing of many materials. The best method that can simulate the clinical situation is the bacterial leakage model. In this study $E$. faecalis bacterial leakage was used. E. faecalis is a gram-positive cocci that can live in short chains, singly or in pairs. It is facultative anaerobes ${ }^{(14)}$. It is considered the most detectable species from oral infections ${ }^{(15)}$. It is also related to different cases of periapical disease and is public in persistent infections ${ }^{(15)}$.

Results reviled that groups irrigated with sodium hypochlorite showed apical bacterial leakage when compared to Biopure MTAD which didn't show any leakage in the $1^{\text {st }}$ week time interval. This could be attributed to the fact that sodium hypochlorite $(\mathrm{NaOCl})$ has excellent antimicrobial action and a capacity to dissolve organic materials, yet it is not completely effective alone to eliminate the smear layer from the canal walls and to prevent the accumulation of hard tissue debris in uninstrumented areas, especially the ramifications ${ }^{(16)}$. While Biopure MTAD, which is a mixture of a tetracycline isomer, citric acid, and a detergent, provides effective elimination of the smear layer with least erosive changes to the dentin surface ${ }^{(17)}$.

The perfect bond of the sealer to the dentin surface is not accomplished without removal of smear layer ${ }^{(18)}$. The interatomic bond which is created at the dentin and smear layer interface cannot withstand polymerization shrinkage during curing of the resin, this bond is approximately $5 \mathrm{MPa}{ }^{(19)}$. Resin will get pulled from the smear layer attached to dentin and lead to leakage.

At 4 weeks period, Biopure MTAD group showed leakage with ActiV GP and AH Plus with gutta percha while EndoRez recorded no apical bacterial leakage. This could be due to the hydrophilic nature of methacrylate resin and the bond formed at the EndoRez sealer and its gutta percha points forming what is called monoblock (a solid mass of obturation material) that allows deeper penetration of the sealer into the dentinal tubules of the internal wall of the root canal ${ }^{(20)}$. While, ActiV Gp group stated that the covering of (GI) filler is non-homogenous and the moisture sensitivity of Glass-ionomer lead to less favorable bonding. In contrast, the scanning electron microscope (SEM) studies indicated that the conventional gutta-percha cones were separated from the AH Plus sealer due to lack of bonding, whereas the resin tags of the sealer remained penetrating dentinal tubules ${ }^{(21)}$. This separation make a break between gutta-percha cones and sealer that leads to microleakage in $\mathrm{AH}$ Plus experimental group.

After 8 weeks, all the specimens in all subgroups markedly increased the mean values of apical bacterial leakage and there was no significant difference found between them. This result is mainly due to the inability of the EndoRez and ActiV Gp to maintain a monoblock for a longer period because of the critical geometry of canals for sealing in addition to the probability for leakage along the sealer - dentin interface ${ }^{(22)}$.

\section{CONCLUSION}

Based on the outcomes of this study EndoRez when used after Biopure MTAD irrigation, apical bacterial leakage was statistically less than ActiV 
GP and AH Plus sealers with gutta percha cones during the first and fourth week's period, where after 8 weeks period all the tested materials showed apical leakage with no statistical difference.

\section{REFERENCES}

1- Nyguen NT: Obturation of the root canal system. In: Cohens S, Burns RC, editors. Pathways of the pulp. 3. St. Louis: CV Mosby Co; 1984.

2- Ingle JI and Taintor JF: Text book of endodontics. Third Edition, 1985.

3- Grossman LI, Oliet S and Delrio CE: Endodontic Practice. Eleventh Edition, 1988.

4- Sevimay S and Kalayci A: Evaluation of apical sealing ability and adaptation to dentine of two resin-based sealers. J Oral Rehab. 32:105-10, 2005.

5- Economides N, Kokorikos I, Kolokouris I, Panagiotis B and Gogos C: Comparative study of apical sealing ability of a new resin-based root canal sealer. J Endod. 30:403-5, 2004.

6- Gogos C, Economides N, Stavrianos C, Kolokouris I and Kokorikos I: Adhesion of a new methacrylate resin-based sealer to human dentin. J Endod. 30: 238-40, 2004.

7- Imai Y and Komabayashi T: Properties of a new injectable type of root canal filling resin with adhesiveness to dentin. J Endod. 29: 20-3, 2003.

8- Zmener O, Pameijer $\mathrm{CH}$ and Macri E: Evaluation of the apical seal in root canals prepared with a new rotary system and obturated with a methacrylate based endodontic sealer: an in vitro study. J Endod. 31:392-5, 2005.

9- Zmener $\mathrm{O}$ and Pameijer $\mathrm{CH}$ : Resin coated gutta-percha cones coupled with a resin-based sealer: a new alternative for filling root canals. Endod Pract. 10:21-5, 2007.

10- Koch K and Brave D: Endodontic synchronicity. Compend Contin Educ Dent. 26:218 -24, 2005.

11- Wolcott J, Himel VT, Powell W and Penney J: Effect of two obturation techniques on the filling of lateral canals and the main canal. J Endod. 23: 632-5, 1997.
12- Torabinejad M, Walton RE and Fouad A: Endodontic principles and practice. 5th ed., Philadelphia, W.B. Sunders Company, 316-338, 2015.

13- Tunga U and Bodrumlu E: Assessment of the sealing ability of a new root canal obturation material. J Endod. 32: 876-8, 2006.

14- Stuart CH, Schwartz SA, Beeson TJ and Owatz CB: Enterococcus faecalis: its role in root canal treatment failure and current concepts in retreatment. J Endod. 32:93-98, 2006.

15- Roc IN, Siqueira JE and Santos KRN: Association of Enterococcus faecalis with different forms of periradicular diseases. J Endod. 30:315-320, 2004.

16- Zehnder M: Root canal irrigants. J Endod. 32: 389-98, 2006.

17- Torabinejad M and Johnson WB: The effect of various concentrations of sodium hypochlorite on the ability of Biopure MTAD to remove the smear layer. J Endod. 29:233-92003.

18- Nakabayashi N: The hybrid layer a resin-dentin composite. Poc Finn Dent Soc. 88:321-9, 1992.

19- Taylor JK, Jeansonne BG and Lemon RR: Coronal leakage effects of smear layer, obturation technique, and sealer. J Endod. 23:508-12, 1997.

20- Tay FR, Loushine RJ, Monticelli F, Weller RN, Breschi L, Ferrari M and Pashley DH: Effectiveness of resin-coated gutta-percha cones and a dual-cured, hydrophilic methacrylate resin based sealer in obturating root canals. J Endod. 31:659- 64, 2005.

21- Shipper G, Orstavik D, Teixeira FB and Trope M: An evaluation of microbial leakage in roots filled with a thermoplastic synthetic polymer-based root canal filling material (Resilon). J Endod. 30:342-7, 2004.

22- Tay FR, Loushine RJ, Lambrecechts P, Weller RN and Pashley DH: Geometric factors affecting dentin bonding in root canals: A theoretical modeling approach. J Endod. $31: 584-9,2005$. 